\title{
Technological Progress of Ultrasound Elastography Based on Shear Waves
}

\author{
Li Qiang \\ Department of BME, Chinese Traditional Medicine Hospital of Tai'an, Tai'an, China \\ Email address: \\ sbk5281@163.com \\ To cite this article: \\ Li Qiang. Technological Progress of Ultrasound Elastography Based on Shear Waves. Clinical Medicine Research. \\ Vol. 9, No. 2, 2020, pp. 42-46. doi: 10.11648/j.cmr.20200902.13
}

Received: October 16, 2019; Accepted: March 25, 2020; Published: April 13, 2020

\begin{abstract}
Elastography aims at assessing tissue elasticity. As a branch of ultrasound elastography (UE), shear-wave elastography is recognized by engineering and clinical fields, particularly fast shear-wave elastography (SWE). Shear-wave elastography (SWE) is a real-time, two-dimensional elastography technology that has emerged in recent years. It is different from Static Elastography, and also different from Transient Elastography and Acoustic Radiation Force Impulse. Based on the fact that the elastic moduli of different tissues are several orders of magnitude greater than the acoustic impedance differences, Elasticity imaging instruments for clinical use have been developed, and has gradually matured. A new stage of technological progress has occurred with SWE. This paper introduces the principle behind the use of elastography and several elastography technologies in clinical application, and then explores methods for fulfilling the promises of this technology: real time and superfast. Furthermore, methods for the generation and detection of Shear Waves are enumerated. These include, for example, the dynamic coherence enhancement technique based on "Mach Waves" and ultra-high-frequency imaging technology for simultaneous transmitting and receiving. Finally, the future development of Shear-wave elastography is discussed. It is believed that with the development of new technologies and new materials, shear wave elastography (SWE) will play an increasingly important role in clinical practice.
\end{abstract}

Keywords: Shear-wave Elastography, Acoustic Radiation Force Impulse, Mach Wave, Ultra High Frequency

\section{Introduction}

Ultrasound diagnostics, including the A-mode (Amplitude), B-mode (Brightness), M-mode (Motion), C-mode (Color), and D-mode (Doppler) ultrasound imaging, have progressed from "point-mode imaging" (A-mode ultrasound), through "linear-mode imaging" (M-mode ultrasound) and "plane-mode imaging" (two-dimensional ultrasound), to "body imaging" (three-dimensional ultrasound) [1]. At present, there is a diagnostic method for measuring tissue stiffness (elasticity) using the principle of ultrasound. Based on the fact that the elastic moduli of different tissues are several orders of magnitude greater than the acoustic impedance differences, Ophir put forward the concept of ultrasonic elastography (UE) in 1991 [2]. Elasticity imaging instruments for clinical use have been developed. In particular, shear wave elasticity imaging (SWEI), also known as E-imaging, has gradually matured. Shear-wave elastography (SWE), used in conjunction with traditional ultrasound imaging, is capable of quantitatively displaying in real-time, tissue elasticity in two dimensions. The value of this technique has been proven in the qualitative diagnosis of breast lesions [3-4]. Dr. James Trotter has also observed that "Shear-wave elastography (SWE) has become an important diagnostic tool for hepatic diseases."

\section{Fundamentals of Elastography}

Elastography aims at assessing tissue elasticity and measuring tissue deformation due to compression. Tissue elasticity is usually expressed as Young's modulus, measured in pascals ( $\mathrm{pa})$ or kilopascals $(\mathrm{kPa})$. The measurement process comprises three steps:

1) The generation of stress within a piece of tissue: stress could be external or internal, and could come from various sources. It could result from static, stable compression, excitation by single-frequency vibrations, or shear waves on the tissue generated by acoustic pulses. 
2) An ultrasound technique is used to measure the tissue displacement caused by stress.

3) The elastic modulus is estimated using the relationship between stress and displacement.

\section{Classification of SWE Techniques}

\subsection{Static SWE Techniques}

Fundamental Principle: When a uniform external pressure or stress (S) is applied to a solid structure, internal deformation in the structure occurs, i.e., strain (e), as shown in Figure 1. Theoretically, elasticity is inversely proportional to strain/displacement when stress is uniformly distributed in the medium [5]. Young's modulus (E) is the ratio between the applied stress and the resulting strain, and this reflects the stiffness of the structure:

$$
\mathrm{E}=\mathrm{s} / \mathrm{e}
$$

Measurement of tissue deformation caused by static, stable compression: A steady and uniform pressure is applied to the body surface of the examinee. Two sets of radio-frequency ultrasound signals (one each for before and after the application of pressure) are collected with an ultrasound probe at the same location. The tissue deformation caused by pressure is calculated and displayed. The clinical application of static SWE has been extensively studied. However, because the exact pressure inside the tissue cannot be assessed, the Young's modulus E cannot be calculated with static elastography. This technique is thus not capable of quantitative evaluation $[6,7]$.

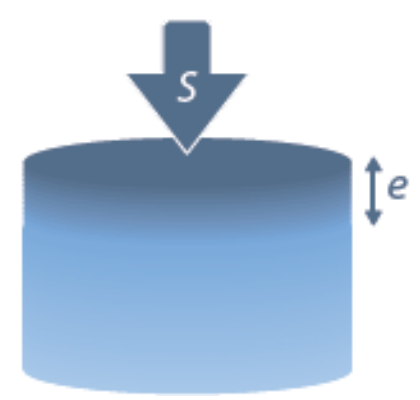

Figure 1. Deformation of a solid elastic body under external forces.

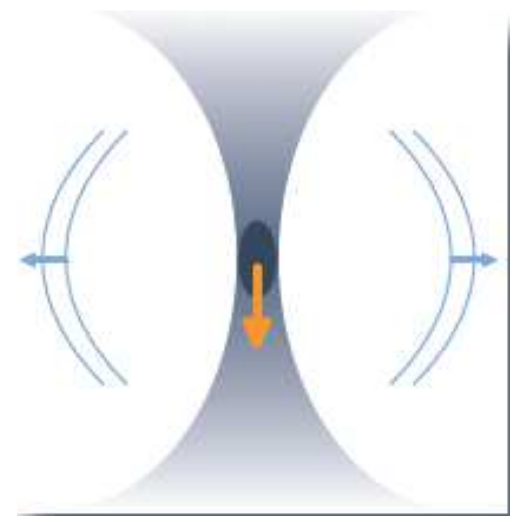

Figure 2. Acoustic radiation force produced by conventional focused acoustic beams.

\subsection{Vibro-acoustography}

Ultrasonic beams of different frequencies are simultaneously focused on the tissue. The targeted area is radiated by alternating radiation forces and gives off acoustic emissions, which are collected to infer the elasticity information of the targeted area [8,9].

This technique gives quantitative evaluation, but not a real-time image. Studies on vibro-acoustography have been performed using the Vivid 7 ultrasound machine of GE [10]. It was also found that vibro-acoustography is more suitable for nuclear magnetic resonance systems [11].

\subsection{Shear-wave Based Technologies}

Basic Principle: Vibration of an acoustic source produces sound waves, which could occur as longitudinal waves, transverse waves, or surface waves. When sound waves are reflected or absorbed along the propagation path, acoustic radiation force is produced. This force causes transverse vibration of the local tissue particles, thereby generating shear waves (Figure 2), one type of transverse wave. Under the influence of the shear modulus, the velocities at which shear waves travel in different soft tissues vary significantly, and the difference could reach several orders of magnitude. Shear wave-based elastography uses pulses to generate shear waves in vivo $[12,13]$, and tissue elasticity is calculated directly by measuring the propagation velocity of the shear waves. The propagation velocity of shear waves in a medium is related to the longitudinal modulus of the biological tissue, which allows for the calculation of elastic modulus of the tissue by measuring shear wave velocity.

Because shear wave propagation is affected by the stiffness of tissue, the Young's modulus (E) is directly related to the propagation velocity of shear waves $(\mathrm{c})$ :

$$
\mathrm{E}=3 \rho \mathrm{v}_{\mathrm{s}}^{2}
$$

where $\rho$ is the density of the tissue $\left(\mathrm{kg} / \mathrm{m}^{3}\right)$, which is generally considered as equivalent to the density of water $\left(1000 \mathrm{~kg} / \mathrm{m}^{3}\right)$. Thus, the elasticity of tissue can be calculated by measuring the propagation velocity $\mathrm{v}$ of shear waves. Three types of techniques are currently used in clinical diagnosis.

\subsubsection{Transient Elastography (IE)}

Shear waves are generated by vibrational excitation when the patient's skin is impacted by low-frequency force from a mechanical source. The propagation velocity of these waves is measured by one-dimensional ultrasound system (about 5 $\mathrm{MHz}$ ). The propagation velocity of shear waves is directly related to elasticity. To account for the subjectivity of manual positioning and operation, 10 measurements are usually taken to calculate the average elasticity. This technique has been successfully applied in clinical practice (FibroScan by Echosens Co., France) [14] for the measurement of liver stiffness and assessment of liver fibrosis. This technique is based on one-dimensional transient elastography. 


\subsubsection{Acoustic Radiation Force Impulse (ARFI) Imaging}

The second technique is ARFI imaging, also known as virtual tough tissue quantification (VTQ) [15]. With ARFI, the tissue is mechanically excited by focusing ultrasound waves from an ultrasonic transducer inside the tested tissue. Small-scale deformations form at specific locations in the tissue, producing shear waves propagating along the transverse direction. These micro-deformations are scanned with high frame-frequency ultrasound beams from the ultrasonic probe. Displacements in the tissue before and after shear wave generation are calculated from radio-frequency data using a cross-correlation algorithm. The propagation velocity of the shear waves in the transverse direction is then determined, from which the elasticity distribution in the biological tissue is quantitatively estimated. This technique could, by and large, serve as the standard for elasticity reconstruction methods across different biological tissues [16].

To generate shear waves of sufficiently high intensity, the acoustic power of ARFI in the focal region needs to be increased to produce greater disturbance. This could cause overheating of the probe and overly large acoustic power [17, 18]. The ACUSON S2000 ultrasound diagnostic device by Siemens is a typical example of ARFI technology. It can be used in conjunction with conventional ultrasound imaging systems to display imaging results, but still fails to break the technical limitations regarding detection depth and acoustic power.

\subsubsection{Shear-wave Elastography (SWE)}

Real-time shear-wave elastography (SWE) integrates two-dimensional form with conventional ultrasound imaging to display quantitatively, real-time tissue elasticity values. The ultrasonic probe of shear-wave elastography (SWE) focuses on deep tissues and generates local acoustic radiation force to produce shear waves in the tissues (Figure 3). Multiple focal points are formed at the same time, which are arranged in an array perpendicular to the patient's body surface, forming a conical shear wave wavefront. The ultrasonic probe scans an entire image along both sides of the focal points. The evolution of shear waves is captured using a fast acquisition technique. The technical design of SWE is shown in Figure 3.

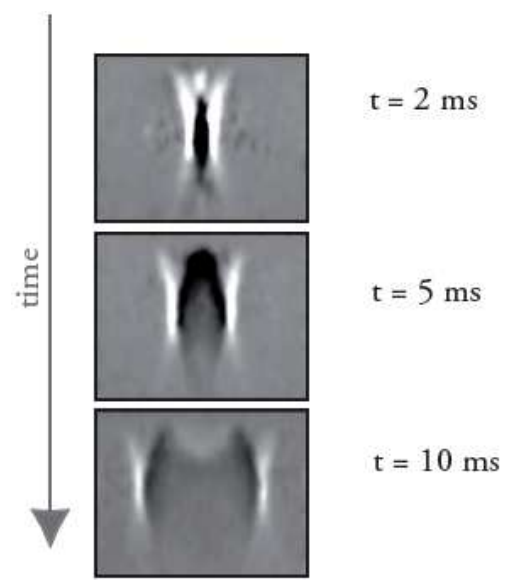

Figure 3. Shear waves generated by acoustic beams focused at the image center.

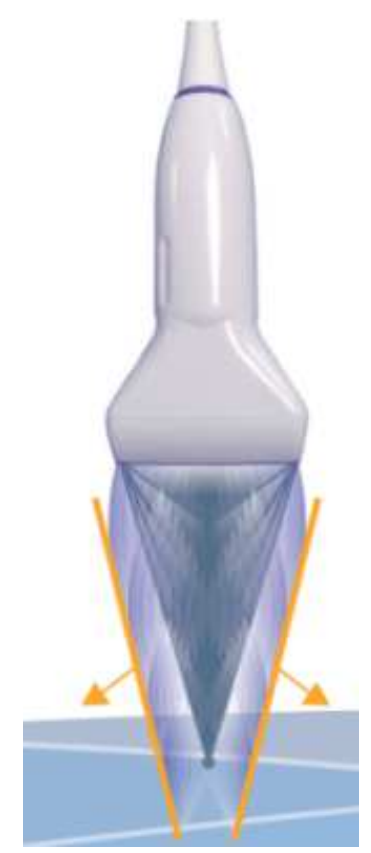

Figure 4. Shear wave amplification and enhancement based on the principle of Mach waves (orange lines).

\section{Realization of SWE}

\subsection{Shear Wave Generation for SWE}

Under ordinary conditions, the shear waves generated by ultrasonic radiation force are only a few microns to tens of microns in amplitude, and can only travel a few millimeters before they disappear. To overcome this disadvantage, technical improvements were made to the ultrasonic probes of SWE equipment. The current technology allows the acoustic beams to focus at different depths in the tissue (Figure 4). The wave sources produced by the focused waves move rapidly in the tissue at a velocity higher than that of the shear waves. The shear waves are enhanced coherently as with Mach waves. A Mach wave is a wavefront formed by a series of perturbations generated by a localized weak perturbation source, which propagate in supersonic airflow. It is an envelope formed by a series of spherical perturbations (Figure 5), and is also called a Mach cone. This probe technology improves the amplitude and propagation distance by 4-8 times. It enhances the amplitude of shear waves and satisfies the requirements for a safety range of acoustic power for ultrasonic probes.

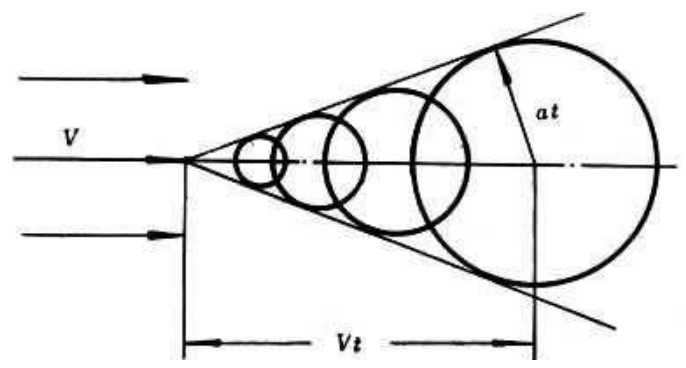

Figure 5. Structure of a Mach wave. 

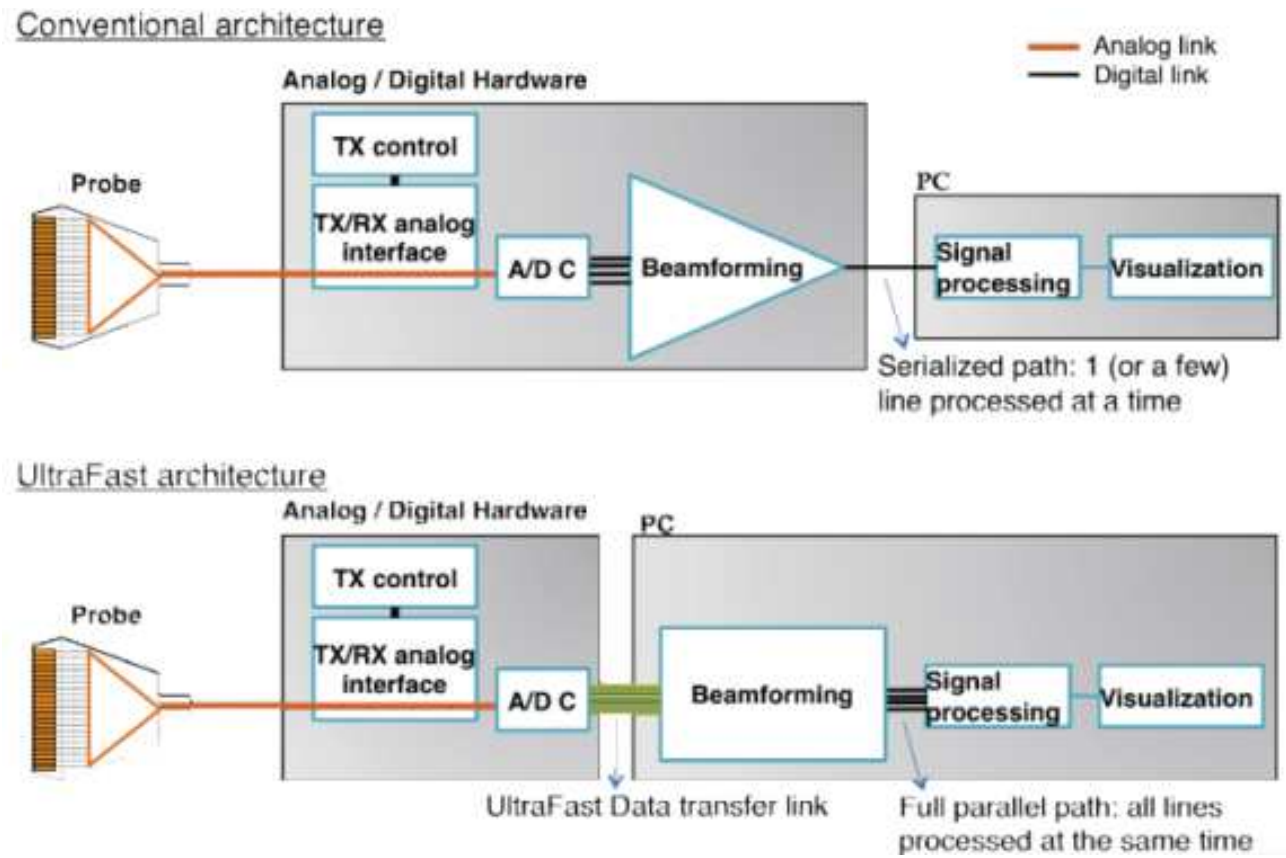

Figure 6. Conventional ultrasound system and UltraFast platform of an Aixplorer system (Supersonic Imagine, France). Acoustic beam formation on the UltraFast platform is accomplished by software. Image computation is parallelized.

\subsection{Shear Wave Detection Technique}

\subsubsection{Using Conventional Ultrasound Imaging}

In conventional ultrasound imaging, the imaging time per image is $T=\left(N_{L} \times 2 D\right) / c$, where $D$ is the imaging depth, $c$ is the acoustic beam propagation velocity, and $\mathrm{N}_{\mathrm{L}}$ is the number of lines in an image. The maximum frame rate is $F_{R}=1 / T=c /$ $\left(\mathrm{N}_{\mathrm{L}} \times 2 \mathrm{D}\right)$.

For instance, an image with a depth of $10 \mathrm{~cm}$ and a width of 256 lines (c is set at $1540 \mathrm{~m} / \mathrm{s}$ ) has a maximum frame rate of $30 \mathrm{~Hz}$.

\subsubsection{Using $S W E$}

The shear waves generated need to be collected and received by an ultrasound system. Shear waves propagate at a velocity of $1-10 \mathrm{~m} / \mathrm{s}$ in tissues and takes approximately 15 milliseconds to pass through the plane of an ultrasound image about $5 \mathrm{~cm}$ in width. At present, the frame rate of ultrasound imaging is $50-100 \mathrm{~Hz}$. Even with the several orders of enhancement in frame rate offered by the multi-line capability of an ultrasound system, the requirements for shear wave acquisition are still not met.

As can be seen from the equation $F_{R}=1 / T=c /\left(N_{L} \times 2 Z\right)$, reducing $\mathrm{N}_{\mathrm{L}}$ can increase the frame rate. If $\mathrm{N}_{\mathrm{L}}$ is 1 (i.e., all lines in the entire image can be calculated in parallel in one transmission), then the maximum frame rate is determined by the time for the ultrasound emitted from the probe to return after traversing the tissue. The UltraFast platform of the Aixplorer system by Supersonic Imagine (France) for example, utilizes plane sound waves. These plane waves are generated by applying delay to the emission elements of the ultrasonic probe, thus acquiring the entire ultrasonic image in one transmission. Under the conventional abdominal imaging condition of $15 \mathrm{~cm}$ depth, the maximum frame rate achieved is
$5,000 \mathrm{~Hz}$, and the maximum frame rate for breast imaging at 4 cm depth is nearly $20,000 \mathrm{~Hz}$. Equipped with ultra-wide beam tracking imaging technology, the color Doppler ultrasound system Resona 7 (Mindray) is able to process in real time all signals in a $0.2-40 \mathrm{~mm}$ wide region and detect shear wave signals at a speed of $10 \mathrm{kHz}$ per frame [19].

\subsubsection{Computation for Acoustic Beam Formation in SWE}

This task is accomplished using a parallel architecture based on a software platform. A technological breakthrough was achieved in data transmission from the acquisition module to the processing unit: radio frequency (RF) signals of data before beamforming are transmitted directly to the computer. The beamforming function of the acquisition module is placed in a personal computer (PC: Figure 6), thus increasing the data transfer rate to several thousand megabytes. Under ultra-high frame rate (UHF) conditions, a specific combination of software and hardware was adopted to collect, receive, and process ultrasound image data at ultra-high speed. The system calculates all lines in a single image simultaneously, and can process at high speed all image data generated at frame rates of thousands of Hertz.

\subsection{Elasticity Calculation and Image Formation in SWE}

Using ultrasound scanning and tissue Doppler imaging techniques with the same ultrasonic probe, any small-scale displacement per unit time in a piece of tissue caused by shear waves can be measured and the propagation velocity of the shear waves can be calculated. This propagation velocity is directly related to elasticity. The Young's modulus $\mathrm{E}(\mathrm{kPa})$ is then derived from Equation (2). The shear wave velocity $(\mathrm{m} / \mathrm{s})$ and elastic modulus of the medium $(\mathrm{kPa})$ are color-coded and displayed. The color-coded image is integrated with a 
gray-scale image (B-mode ultrasound) containing anatomical information to form a two-dimensional image that displays quantitatively in real time the elasticity characteristics of the tissue.

\section{Technical Prospects of Shear-wave Elastography (SWE)}

Technologies in this area are constantly advancing by leaps and bounds. As a branch of ultrasound elastography (UE), shear-wave elastography is recognized by engineering and clinical fields, particularly fast shear-wave elastography (SWE). However, theoretical and technical difficulties still exist. Improvements are urgently needed on the anisotropy, nonlinearity, and imaging depth of shear wave elastography in tissues. The new BI-RADS report published in 2013 expresses caution regarding UE. The clinical effectiveness of this technology is not explicitly acknowledged in this report. It is believed that with the development of new technologies and new materials, shear wave elastography (SWE) will play an increasingly important role in clinical practice.

\section{References}

[1] Chen JM, Ji JH, Li GD. Fundamental principles of ultrasound diagnostic instruments and applications of new technologies. Journal of Medical Equipment Information 2006; 21 (12): 28-30.

[2] Ophir J, Céspedes I, Ponnekanti H, Yasdi Y, Li X. Elastography: A quantitative method for imaging the elasticity of biological tissues. Ultrasound Imaging 1991; 13: 111-134.

[3] Tanter M, Bercoff J, Athanasiou A, Deffieux T, Gennisson J-L, Montaldo G, Muller M, Tardivon A, Fink M. Quantitative assessment of breast lesion viscoelasticity: Initial clinical results using supersonic shear imaging. Ultrasound In Medicine and Biology 2008; 34 (9): 1373-1386.

[4] Athanasiou A, Tardivon A, Tanter M, Sigal-Zafrani B, Bercoff J, Deffieux T, Gennisson JL, Fink M, Neuenschwander S. Breast lesions: quantitative elastography with supersonic shear imaging-preliminary results. Radiology 2010 Jul; 256 (1): 297-303.

[5] Li JL, Shi XQ. Advances in the application of ultrasound elastography in the evaluation of breast diseases. Journal of International Medical Devices 2015; 21 (2): 38-40.

[6] Svensson WE, Amiras D. Ultrasound elasticity imaging. Breast Cancer Online 2006; 9: e24 (7 pages). Cambridge University Press. Barr RG. Clinical applications of a real time elastography technique in breast imaging. Proceedings of the 5th International Conference on Ultrasonic Measurement and Imaging of Tissue Elasticity 2006: 112.

[7] Locatelli M, Chervesani R, Rizzatto G. Real-time ultrasound Elastography: diagnostic tool or electronic gadget? European Radiology 2005; 15 supplement 1 (ECR 2005, book of abstracts) abstract B-0255: 139.

[8] Muthupillari R, Lomas DJ, Rossman PJ, Greenleaf JF, Manduca A, Ehman RL. Magnetic resonance elastography by direct visualization of propagating acoustic strain wave. Science 1995; 269: 1854-1856.

[9] Parker KJ, Lerner RM. Sono elasticity of organs: Shear waves ring a bell. Journal of Ultrasound in Medicine 1992; 11: 387-392.

[10] Huang S. Ultrasound elastography and its clinical application. Journal of International Medical Devices, 201; 21 (2): 24-28.

[11] Sinkus R, Tanter M, Xydeas T, Catheline S, Bercoff J, Fink M. Visco elastic shear properties of in vivo breast lesions measured by MR elastography. Magnetic Resonance Imaging 2002; 23 (2): 159-165.

[12] Bercoff J, Tanter M, Fink M. Supersonic shear imaging: A new technique for soft tissues elasticity mapping. IEEE Transactions on Ultrasonics, Ferroelectrics, and Frequency Control 2004; 51 (4): 396-409.

[13] Sarvazyan AP, Rudenko OV, Swanson SD, Fowlkes JB, Emelianov SY. Shear wave elasticity imaging: A new ultrasonic technology of medical diagnostic. Ultrasound in Medicine and Biology 1998; 20: 1419-1436.

[14] Sandrin L, Fourquet B, Hasquenoph JM, Yon S, Fournier C, Mal F, Christidis C, Ziol M, Poulet B, Kazemi F, Beaugrand M, Palau R. Transient elastography: a new noninvasive method for assessment of hepatic fibrosis. Ultrasound in Medicine and Biology 2003 Dec; 29 (12): 1705-1707.

[15] Cui GH, Yang $\mathrm{Z}$ et al. Factors influencing acoustic radiation force impulse imaging: a discussion based on animal models [J]. China Medical Devices, 2012; 27 (10): 141-142.

[16] Sun WL, Yan BG, Ma L. Ultrasound elastography and its application. Journal of Progress in Modern Biomedicine, 2007; 7 (9): 1411-1413.

[17] IEC 60601-2-37: 2001 + Amendment 1: 2004 + Amendment 2: 2005: Medical electrical equipment-Part 2-37: Particular requirements for the safety of ultrasonic medical diagnostic and monitoring equipment.

[18] Nightingale KR, Soo MS, Nightingale RW, Trahey GE. Acoustic radiation force impulse imaging: in vivo demonstration of clinical feasibility. Ultrasound in Medicine and Biology 2002; 28 (2): 227-235.

[19] Li SS, Li YD. STE elastography-a new solution for ultrasound elastography. Journal of International Medical Devices, 2016; 22 (9): 53-55. 